\title{
Small-Angle Neutron Scattering Study of the Structural Relaxation of Elongationally Oriented, Moderately Stretched Three-Arm Star Polymers
}

Mortensen, Kell; Borger, Anine L.; Kirkensgaard, Jacob J. K.; Huang, Qian; Hassager, Ole; Almdal, Kristoffer

Published in:

Physical Review Letters

Link to article, DOI:

10.1103/PhysRevLett.127.177801

Publication date:

2021

Document Version

Publisher's PDF, also known as Version of record

Link back to DTU Orbit

Citation (APA):

Mortensen, K., Borger, A. L., Kirkensgaard, J. J. K., Huang, Q., Hassager, O., \& Almdal, K. (2021). Small-Angle Neutron Scattering Study of the Structural Relaxation of Elongationally Oriented, Moderately Stretched ThreeArm Star Polymers. Physical Review Letters, 127(17), [177801].

https://doi.org/10.1103/PhysRevLett.127.177801

\section{General rights}

Copyright and moral rights for the publications made accessible in the public portal are retained by the authors and/or other copyright owners and it is a condition of accessing publications that users recognise and abide by the legal requirements associated with these rights.

- Users may download and print one copy of any publication from the public portal for the purpose of private study or research.

- You may not further distribute the material or use it for any profit-making activity or commercial gain

- You may freely distribute the URL identifying the publication in the public portal 


\title{
Small-Angle Neutron Scattering Study of the Structural Relaxation of Elongationally Oriented, Moderately Stretched Three-Arm Star Polymers
}

\author{
Kell Mortensen $\odot^{*}$ and Anine L. Borger \\ Niels Bohr Institute, University of Copenhagen, 2100 Copenhagen, Denmark \\ Jacob J. K. Kirkensgaard $\odot$ \\ Niels Bohr Institute and Dept. Food Science, University of Copenhagen, 2100 Copenhagen, Denmark \\ Qian Huang () \\ Polymer Research Institute, Sichuan University, 610065 Chengdu, China \\ Ole Hassager® \\ Department of Chemical and Biochemical Engineering, Technical University of Denmark, 2800 Kongens Lyngby, Denmark \\ Kristoffer Almdal@ \\ Department of Chemistry, Technical University of Denmark, 2800 Kongens Lyngby, Denmark
}

(Received 22 June 2021; accepted 21 September 2021; published 18 October 2021)

\begin{abstract}
We present structural relaxation studies of a polystyrene star polymer after cessation of high-rate extensional flow. During the steady-state flow, the scattering pattern shows two sets of independent correlations peaks, reflecting the structure of a polymer confined in a fully oriented three-armed tube. Upon cessation of flow, the relaxation constitutes three distinct regimes. In a first regime, the perpendicular correlation peaks disappear, signifying disruption of the virtual tube. In a second regime, broad scattering arcs emerge, reflecting relaxation from highly aligned chains to more relaxed, still anisotropic form. New entanglements dominate the last relaxation regime where the scattering pattern evolves to a successively elliptical and circular pattern, reflecting relaxation via reptation.
\end{abstract}

DOI: 10.1103/PhysRevLett.127.177801

Viscoelastic properties of polymeric liquids constitute a central research subject in polymer physics. The flow and deformation of macromolecules are a ubiquitous phenomena in nature as well as in industrial processing, and are governed by molecular chemistry, molecular architecture and mechanical treatments. Architecturally complex polymers attract increased attention due to the remarkable properties. Star polymers, for example, have been the subject of several studies [1-13].

The dynamics of polymeric materials under small deformations are well described by a slightly extended version of the tube model originally proposed and described by de Gennes as well as Doi and Edwards [14-21]. However, the dynamics of strongly deformed and branched polymers can be expected to be much more complex and the validity of the tube model is highly challenged [9,22-24].

In a recent study, prior for the present work, we studied the deformed structure of a three-armed star polymer during fast extensional flow [1]. We showed unambiguously that this system remains to have conformation characteristics of a tube. Immediately after cessation of extensional flow, neutron scattering experiments showed the characteristics of a fully aligned three-armed tube. Figure 1 reviews the schematic molecules as visualized in the relaxed state (a) and in the oriented, slightly stretched state during flow (as measured immediately after cessation of flow) (b) [1]. In the relaxed state, the polymer configuration is in agreement with the structure factor calculated based on the random phase approximation, RPA. The molecular configuration in the stretched state corresponds to that of a polymer confined in a fully oriented and slightly extended three-armed tube with two arms right next to each other.

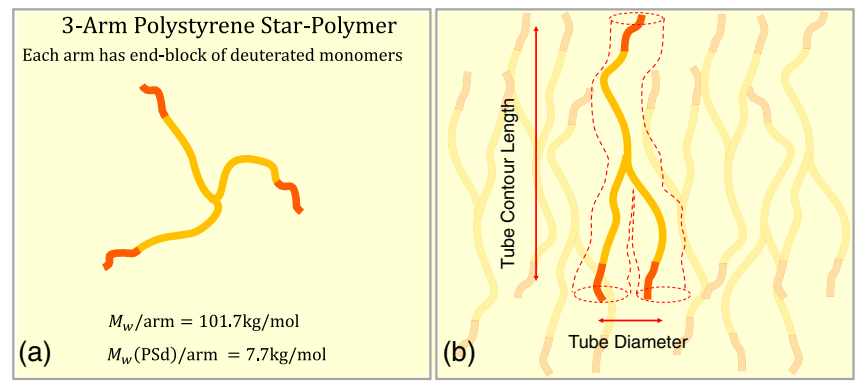

FIG. 1. Molecular characteristics of the three-arm star-copolymer (a), and a schematic illustration of the molecular configuration in the stretched state, immediately after cessation of flow (b) (for details, see Ref. [1]). 
Huang et al. made rheological relaxation experiments after cessation of extensional flow on a three-arm starpolymer melt equivalent to that discussed in this Letter [4]. The measurements were made under exact similar temperature and flow conditions as those of the present study. Huang et al. suggested that the relaxation dynamics fall into three regimes. At very short times, region I $(<15 \mathrm{~s})$, it was found that the three-arm star relaxes identically to a linear polymer with molar mass equal two times the arm length. At intermediate times, region II (15-500 s), the star polymer deviates slightly from the linear polymer. For regions I and II, it was speculated that the arms relax independently. At long times, the terminal region III $(>500 \mathrm{~s})$, the relaxation behavior is in agreement with orientational relaxation via reptation [15,25] (for a detailed discussion, we refer to the work of Huang et al. [4]).

In this study, we present experimental structural studies of the relaxation dynamics of the symmetric three-arm star polymer with molecular characteristics similar to that used by Huang et al. in their rheological study [4]. The only difference is that for the structural studies we used threearm stars with deuterium labeling at the end of each arm, to make contrasts for the neutron diffraction. We find that the structural relaxation falls into three surprisingly distinct time regimes with completely different characteristics, thereby supporting the conclusion from rheology. The initial configuration observed right after cessation of flow, corresponds to that of a fully extended three-armed virtual tube [1]. The initial relaxation (I) is dominated by disruption of the tube. In the second relaxation regime (II) the arms relax from being confined in the aligned, cylindrical shaped tubes to a more relaxed, still anisotropic structure and form new entanglements. In the final relaxation regime (III), the chain relaxation is dominated by orientational relaxation and approaches the completely relaxed structure. Related studies were previously made on end-labeled $\mathrm{H}$-shaped molecules [26], showing unique anisotropic SANS pattern, but different from our observations. Their data include also a four-peak pattern, which, however, approached a butterfly symmetry. The observations could not be explained in the simple RPA approach, but it was shown that elastic fluctuations and inhomogeneities play an important role [27].

The polymer (PS- $b$-PS $d)_{3}$ was a three-armed polystyrene star, deuterium labeled at the very end of each arm. The deuterated blocks were chosen to be relatively small to get precise and easily accessible information on the star polymer deformation, even though the small PS $d$ blocks cause rather weak scattering and thereby somewhat noisy data. The PS-PS $d$ block copolymer arms have molar mass $M_{w}=101.7 \mathrm{~kg} / \mathrm{mol}$ with a mass fraction of deuterated polystyrene $f=0.075$. The resulting three-arm block copolymer star has a molar mass of $309.1 \mathrm{~kg} / \mathrm{mol}$ and polydispersity 1.30. Details of the synthesis is given in Ref. [1].
The Kuhn segment length of polystyrene is $b=18 \AA$, the entanglement molar mass is $M_{e}=16.6 \mathrm{~kg} / \mathrm{mol}$ and the tube diameter is $a=85 \AA$ [28]. With the molar mass $M_{w}=$ $101.7 \mathrm{~kg} / \mathrm{mol}$ for each arm, the number of entanglements is $Z \approx 6$, and the equilibrium end-to-end length is $R_{\circ}(1 \mathrm{arm}) \approx 210 \AA$ [1]. The number of Kuhn segments in each arm is $N_{\text {arm }}=\left(R_{\circ} / b\right)^{2}=135$, and the total number of segments in the star polymer is $N=3 N_{\text {arm }}=405$.

The samples were exposed to extensional flow at $T=$ $125^{\circ} \mathrm{C}$ with a strain rate of $\dot{\epsilon}=0.06 \mathrm{~s}^{-1}$, using a VADER 1000 instrument (Rheo Filament ApS). The Rouse time of a linear chain of the same molar mass is $450 \mathrm{~s}$ at $125^{\circ} \mathrm{C}$ [4]. Since the applied strain rate is significantly greater than the inverse Rouse time, the star molecules are expected to be highly oriented and nonlinearly stretched, as experimentally verified [1]. Steady state flow conditions are established beyond a Hencky strain of $\epsilon=3$ [29], which is the Hencky strain applied in the present study.

Samples were quenched from different stages of relaxation at $125^{\circ} \mathrm{C}$. The quench-cooling rate was about $10 \mathrm{~K} / \mathrm{s}$. Since the glass transition temperature is $105^{\circ} \mathrm{C}$, the melt solidifies in a time much shorter than the Rouse time as shown by Kirkensgaard et al. [24]. The molecular configurations measured in the solid samples can therefore be assumed to be identical to those in the melt at the time of quench.

The structural studies were performed by small-angle neutron scattering (SANS) using the Quokka instrument at ANSTO, Australia. Two instrumental settings were applied, both with neutrons with wavelength $5 \AA$ and $10 \%$ wavelength resolution and the collimation defined by a source diameter of $50 \mathrm{~mm}$ and a pinhole in front of the sample of $10 \mathrm{~mm}$. One instrumental setting had $10 \mathrm{~m}$ collimation length and $5 \mathrm{~m}$ sample-to-detector distance, giving a $q$ range equal to $0.01-0.15 \AA^{-1}$; the second setting used collimation length and sample-to-detector distance both equal to $14 \mathrm{~m}$, giving a $q$ range of $0.004-0.06 \AA^{-1}$, where $q$ is the scattering vector.

The experimental 2D SANS data are shown in Fig. 2. The 2D SANS data were corrected for background and detector sensitivity, using standard methods [30]. The absolute intensities are, however, still somewhat uncertain due to uncertainty in the absolute amount of samples in the beam. The resulting contour diagrams given in Fig. 2 are plotted using the same color scale and can accordingly be compared with respect to both intensity and geometrical pattern. The lower row shows a schematic illustration of the findings to clarify the discussion in the text. Figure 3 shows the time evolution of the $q$ values of the peak-center parallel and perpendicular to flow direction, derived from the 2D patterns in Fig. 2. The data show three distinct regimes of different scattering pattern characteristics, equivalent to the conclusion from the rheological study [4].

Figures 2(a) and 2(i) are data obtained immediately after cessation of the elongational flow. The data show a very 


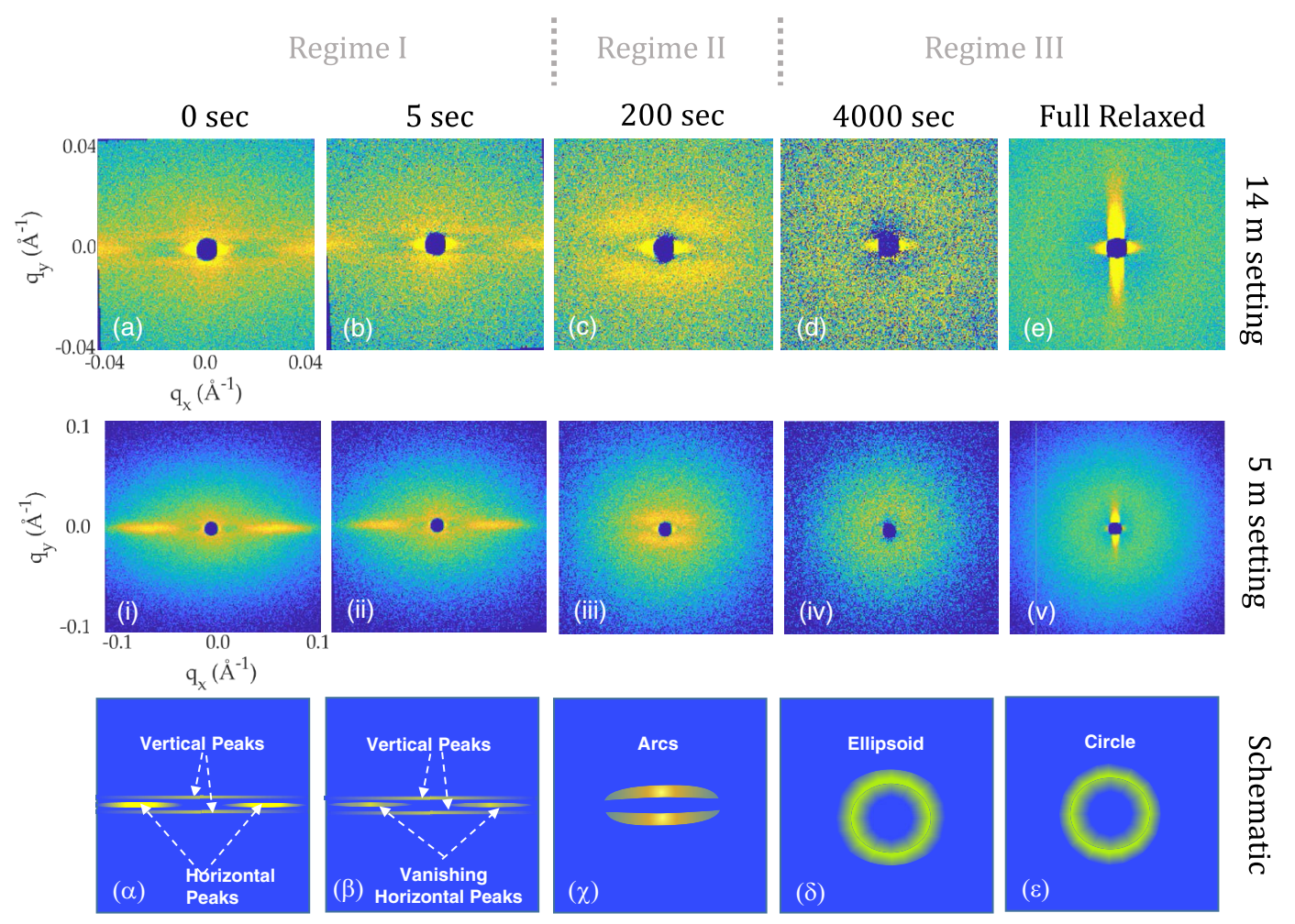

FIG. 2. Experimental 2D SANS patterns of star-polymer system, as obtained at different times after cessation of flow-induced elongational stretching (vertically). The upper and middle rows are data obtained in the 14 and $5 \mathrm{~m}$ instrument configuration, all on the same contour color scale. The last row shows a schematic illustration of the findings to clarify the discussion in the text. The regimes I, II, and III refer to distinct different SANS characteristics reflecting the different structural dominance during the relaxation.

unique and characteristic pattern, which was the focus of the preceding Letter [1]. The SANS pattern is dominated by two sets of correlation peaks: one pair at the horizontal axis around $q_{\perp}^{*}= \pm 0.06 \AA^{-1}$ and another pair centered at the vertical axis around $q_{\|}^{*}= \pm 0.005 \AA^{-1}$. Both sets of correlation peaks have very large horizontal dispersions, i.e., perpendicular to the flow direction, while being highly

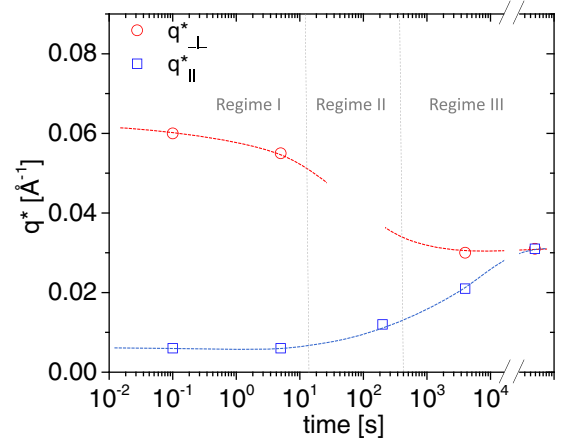

FIG. 3. Peak positions derived from SANS study of starpolymer system during relaxation after cessation of high-flowinduced stretching, showing the $q^{*}$ values as observed perpendicular (circles) and parallel (squares) to original flow direction. The dotted lines are a guide to the eye. In region II, we were not able to detect any peaks on the axis perpendicular to the flow direction. confined vertically parallel to the flow. (The index “*”" on $q$ is used to point to the $q$ values measured at the center value of the horizontal streaks.) It is interesting to note that the horizontal and vertical peaks do not merge, i.e., they are not part of a common correlation ring as might have been expected to emerge from "deformation" of the relaxed pattern. The SANS patterns have additional horizontal scattering near the beamstop, which is attributed to reflection from the thin, cylinder shaped samples.

The origin of the unique scattering pattern right after cessation of flow [Figs. 2(a) and 2(i)] was discussed in the preceding Letter [1]. The two peaks centered on the vertical axis $\left(q_{\|}^{*}= \pm 0.005 \AA^{-1}\right)$ having character of horizontal streaks reflect correlation between two deuterated end blocks parallel to the flow, one diblock copolymer arm in positive and one in negative direction, as sketched in Fig. 1(b). The theoretical length of a fully aligned tube of the star polymer is given by the sum of the tube lengths of two arms, $2 L_{\circ}=2 Z a=1040$. This value is, with our experimental resolution, close to the experimental value $2 \ell$ obtained from the vertical peaks of $q_{\|}^{*}= \pm 0.005 \AA^{-1}$, giving $2 \ell=2 \pi /\left|q_{\|}^{*}\right|=1300 \AA$. The slightly larger $2 \ell$ value indicates molecular stretching. (The length of a star polymer with all Kuhn segments aligned would be $2 N_{\mathrm{arm}} b=4860 \AA$.) 


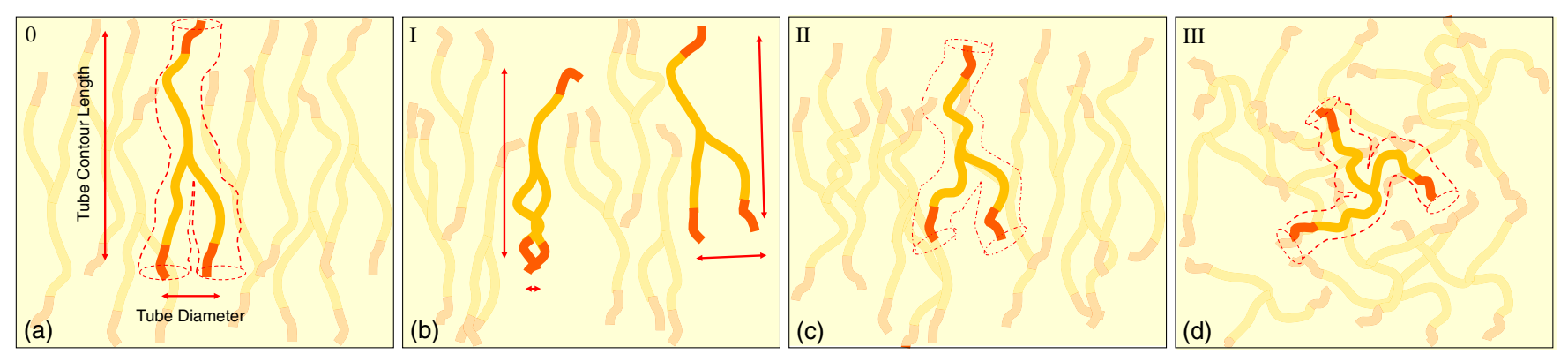

FIG. 4. Schematic figure of the aligned, slightly stretched star block copolymer. (a) Represents the fully extended three-armed virtual tube with two of the tube arms positioned right next to each other. (b) Represents the initial relaxation I, where the structure factor is dominated by relaxation of the arms without a confining tube, i.e., weakening and loosing the perpendicular correlation peaks. (c) Represents regime II with relaxation dominated by chain relaxation parallel to strain and reestablishing entanglements. (d) Represents a polymer that is deformed within the linear regime of stretching following the dynamics of the reptation model.

The two peaks centered on the horizontal $q_{\perp}$ axis with center values $q_{\perp}^{*}= \pm 0.06 \AA^{-1}$ correspond to a distance of the order of $100 \AA$, i.e., close to the tube diameter $a=85 \AA$, suggesting that the two arms make up individual tubes right next to each other. Our interpretation of the experimental scattering pattern was thus that of a threearmed fully aligned tube confining the polymer chains [1], as sketched in Fig. 1(b).

Even though our proposed tube picture may be somewhat naive, it provides a very good account for the experimental findings, not only during flow as discussed previously [1], but also during relaxation. Right after cessation of flow, the scattering pattern shows that the star polymers remain in a configuration as being confined in a virtual three-armed tube, as sketched in Fig. 4(a). While this fully aligned tube confinement is a consequence of the fast flow, one may speculate that the polymer in this extended configuration in reality has only a limited physical counterpart in terms of entanglements. The vertical and the horizontal scattering peaks persist both for some time. The intensity and the $q$ value of the correlation peaks in direction of flow (vertical axis) remain within statistics unchanged in region $I$. The intensity of the horizontal peaks, on the other hand, decreases. This signifies that the initial relaxation is dominated by dynamics of the ends of the block-copolymer arms. Consequently, there is no longer a characteristic distance between the two deuterated end blocks [Fig. 4(b)], and the correlation peaks vanish. This initial relaxation dominated by the ends of the arms explains very well the rheological findings in regime I, where the three-armed star polymer behaves similarly to the linear polymer [4], i.e., dominated by the ends of the arms and not affected by the branch point. The scattering intensity of the horizontal peaks has completely vanished when reaching region II and the $q$ value of the vertical peak has increased, signifying the absence of the chain stretched beyond the Rouse time.

The scattering pattern of region II, $15 \mathrm{~s}<\tau<500 \mathrm{~s}$, is characterized by two broad arcs centered on the vertical axis, as shown in Figs. 2(c) and 2(iii). The $q$ value, $q_{\|}^{*}$, is increased relative to the streaks observed immediately after cessation of flow. The arclike scattering pattern centered around the vertical axis shows that there is no well-defined correlation distance between the two deuterated blocks oriented in the same direction relative to the flow. The significantly broadened arclike peaks with increased $q_{\|}^{*}$ value reflect that the polymer arms are no longer restricted to the tube orientation parallel to the flow, but are flexible oriented around the flow direction with varying distance and orientation between the two deuterated blocks aligned in the opposite direction relative to flow. New entanglements may be formed among the flexible polymer arms, as sketched in Fig. 4(c).

Approaching the last relaxation regime III, $\tau>500 \mathrm{~s}$, the scattering arcs of regime II approaches a complete correlation ring [Figs. 2(d) and 2(iv)]. During the relaxation in regime III the scattering pattern relaxes from an elliptical to a circular correlation ring [Figs. 2(e) and 2(v)] in agreement with a totally relaxed three-arm star-blockcopolymer sketched in Fig. 4(d). The relaxed SANS pattern has an intensity versus $q$ behavior which is in perfect agreement with the RPA-structure factor of a relaxed star polymer [1]. The structural findings are too limited to provide details on the relaxation mechanism in regime III, but the scattering data are in agreement with the relaxation mechanism predicted in the tube model, dominated by orientational relaxation via arm-length fluctuation and reptation [4].

The scattering pattern of the fully relaxed sample is unfortunately affected by artificial small-angle scattering near the beamstop, which arises from the sample itself, but likely related to cracks [1]. Such cracks seem to be a general property for polystyrene samples that are fully relaxed after being exposed to high extensional flow rates, and will be the subject of a forthcoming study. The other measured samples of the present study, which have not been fully relaxed, have no similar problems with cracks. Fortunately, the crack-based small-angle scattering does 
not disturb the structural characteristics relevant for the star-polymer conformation.

In summary, we have made a "real-time" structural relaxation study of a three-armed polystyrene star polymer. Upon exposure to large extensional flows, the star polymer constitutes a highly stretched structure that mimics that of a fully aligned, slightly extended three-armed tube, with one arm in one direction and two arms in the opposite direction relative to the direction of the flow. Upon cessation of flow, the relaxation constitutes three regimes with distinct and different characteristics. In the initial relaxation regime the well-defined distance between the two end blocks stretched in the same direction relative to flow disappears. After cessation of flow the ends of the polymer arms relax and the perpendicular scattering peaks vanish. The scattering emerges into a pattern of exclusively parallel correlation peaks, which when reaching regime II changes character from the highly confined streaks to broad arclike correlation peaks, reflecting more relaxed chains and likely new entanglements. This dominates the last part of the relaxation mode, regime III, where the scattering pattern evolves from the two arcs to an elliptical pattern, and further on to a circular correlation ring in accordance with the RPA structure of a completely relaxed star polymer.

This work was funded from the Independent Research Fund Denmark, Technology and Production (4005-00112), the Velux Foundation, the Aage and Johanne Louis-Hansen Foundation, and the Danish Research Infrastructure via DANSCATT. Andriy Dorokhin is acknowledged for sample synthesis, and Christopher J. Garvey is acknowledged for neutron scattering assistance. ANSTO are acknowledged for neutron beam time at Quokka.

*kell@nbi.ku.dk

[1] K. Mortensen, A. L. Borger, J. J. K. Kirkensgaard, C. J. Garvey, K. Almdal, A. Dorokhin, Q. Huang, and O. Hassager, Phys. Rev. Lett. 120, 207801 (2018).

[2] Y. Matsumiya and H. Watanabe, Prog. Polym. Sci. 112, 101325 (2021).

[3] T. Kinsey, E. U. Mapesa, W. Wang, K. Hong, J. Mays, S. M. Kilbey, and J. Sangoro, Macromolecules 54, 183 (2021).

[4] Q. Huang, S. Agostini, L. Hengeller, M. Shivokhin, N. J. Alvarez, L. R. Hutchings, and O. Hassager, Macromolecules 49, 6694 (2016).

[5] X. Xu and J. Chen, J. Chem. Phys. 144, 244905 (2016).

[6] N. H. Aloorkar, A. S. Kulkarni, R. A. Patil, and D. J. Ingale, Int. J. Pharm. Sci. Nanotechnol. 5, 1675 (2012).

[7] M. Ripoll, R. G. Winkler, and G. Gompper, Phys. Rev. Lett. 96, 188302 (2006).
[8] J. G. H. Cifre, R. Pamies, M. C. L. Martinez, and J. G. de la Torre, Polymer 46, 6756 (2005).

[9] T. C. B. McLeish, Adv. Phys. 51, 1379 (2002).

[10] D. Vlassopoulos, G. Fytas, T. Pakula, and J. Roovers, J. Condens. Matter Phys. 13, R855 (2001).

[11] X. Ye and T. Sridhar, Macromolecules 34, 8270 (2001).

[12] A. Dondos, D. Papanagopoulos, P. G. de Gennes, and F. Brochard-Wyart, Macromol. Theory Simul. 8, 147 (1999).

[13] G. S. Grest, L. J. Fetters, J. S. Huang, and D. Richter, Star Polymers: Experiments, Theory, and Simulation, edited by I. Prigogine and S. A. Rice, Advances in Chemical Physics, Vol. XCIV (John Wiley \& Sons, New York, 1996), ISBN: 0-471-14324-3.

[14] P. G. de Gennes, J. Chem. Phys. 55, 572 (1971).

[15] M. Doi and S. F. Edwards, The Theory of Polymer Dynamics (Clarendon Press, Oxford, 1988), ISBN: 9780198520337.

[16] P. Schleger, B. Farago, C. Lartigue, A. Kollmar, and D. Richter, Phys. Rev. Lett. 81, 124 (1998).

[17] V. G. Sakai, C. Alba-Simionesco, and S.-H. Chen, Dynamics of Soft Matter, Neutron Applications (Springer, New York, 2012), ISBN: .

[18] D. J. Read and T. C. B. McLeish, Phys. Rev. Lett. 79, 87 (1997).

[19] A. E. Likhtman and T. C. B. McLeish, Macromolecules 35, 6332 (2002).

[20] W. Pyckhout-Hintzen, S. Westermann, A. Wischnewski, M. Monkenbusch, D. Richter, E. Straube, B. Farago, and P. Lindner, Phys. Rev. Lett. 110, 196002 (2013).

[21] Z. Wang, C. N. Lam, W.-R. Chen, W. Wang, J. Liu, Y. Liu, L. Porcar, C. B. Stanley, Z. Zhao, K. Hong, and Y. Wang, Phys. Rev. X 7, 031003 (2017).

[22] M. Zamponi, M. Kruteva, M. Monkenbusch, L. Willner, A. Wischnewski, I. Hoffmann, and D. Richter, Phys. Rev. Lett. 126, 187801 (2021).

[23] O. Hassager, K. Mortensen, A. Bach, K. Almdal, H. K. Rasmussen, and W. Pyckhout-Hintzen., Rheol. Acta 51, 385 (2012).

[24] J. J. K. Kirkensgaard, L. Hengeller, A. Dorokhin, Q. Huang, C. J. Garvey, K. Almdal, O. Hassager, and K. Mortensen, Phys. Rev. E 94, 020502(R) (2016).

[25] T. C. B. McLeish, Adv. Phys. 51, 1379 (2002).

[26] M. Heinrich, W. Pyckhout-Hintzen, J. Allgaier, D. Richter, E. Straube, D. J. Read, T. C. B. McLeish, D. J. Groves, R. J. Blackwell, and A. Wiedenmann, Macromolecules 35, 6650 (2002)

[27] D. J. Read, Macromolecules 37, 5065 (2004).

[28] L. J. Fetters, D. J. Lohsey, and R. H. Colby, Physical Properties of Polymers Handbook, 2nd ed., edited by James E. Mark (Springer, New York, 2007), Vol. 3, p. 25, ISBN: 978-0-387-31235-4.

[29] L. Hengeller, Q. Huang, A. Dorokhin, K. Almdal, N. J. Alvarez, and O. Hassager, Rheol. Acta 55, 303 (2016).

[30] I. W. Hamley, Small-Angle Scattering: Theory, Instrumentation, Data, and Applications (John Wiley \& Sons, New York, 2021), ISBN: 978-1-119-76830-2. 